\title{
Constitutional DNA
}

National Cancer Institute ( $\mathrm{NCl})$

\section{Source}

National Cancer Institute (NCI). Constitutional DNA.

Constitutional DNA refers to tissue derived from reproductive cells (egg or sperm) that become incorporated into the DNA of every cell in the body of the offspring. A germline mutation may be passed from parent to offspring. Also called germline DNA. 